
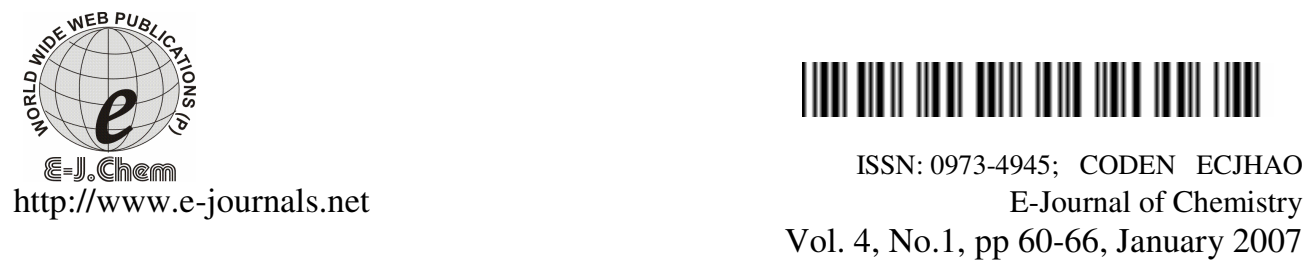

\title{
Studies on Synthesis of Pyrimidine Derivatives and their Pharmacological Evaluation
}

\author{
T. A. NAIK and K. H. CHIKHALIA* \\ Department of chemistry, \\ Veer Narmad South Gujarat University, \\ Surat-395 007, Gujarat, India \\ E-mail: drtaralnaik@yahoo.com
}

Received 14 August 2006; Accepted 3 October 2006

\begin{abstract}
: 1,3,4-oxadiazoles were associated with broad spectrum of biological activities including antituberculosis, anticonvulsant, antiinflammatory, insecticidal, antifungal, analgesic and antitumor properties. Morpholine derivatives find their wide spectrum of antimicrobial activity and exhibit anthelmintic, bactericidal and insecticidal activity. Pyrimidine derivatives are also reported to possess antibacterial, antimicrobial, antifungal, anticancer and anticonvulsant activities. Encouraged by this observations we decided to synthesised novel pyrimidine derivatives.
\end{abstract}

Keywords: Oxadiazoles, Pyrimidine Derivatives, Synthesis

\section{Introduction}

The biological significance of the pyrimidine derivatives has led us to the synthesis of substituted pyrimidine. As pyrimidine is a basic nucleus in DNA \& RNA, it has been found to be associated with diverse biological activities. ${ }^{1}$ The synthesis of substituted pyrimidine and many detailed reviews have been appeared. ${ }^{2,3}$.

The nitrogen containing fragment may be an amidine, urea, thiourea or guanidine and acetyl acetone serves as an excellent illustrative example in that it readily undergoes reaction with formamidine, ${ }^{4}$ guanidin, ${ }^{5}$ urea, ${ }^{6}$ or thiourea ${ }^{7}$ to produce the corresponding 4,6-dimethyl pyrimidine.

Pyrimidines and their derivatives are considered to be important for drugs and agricultural chemicals. Pyrimidine derivatives possess several interesting biological 
activities such as antimicrobial ${ }^{8}$, antitumour ${ }^{9}$ and antifungal activities ${ }^{10}$. Many Pyrimidine derivatives are used for thyroid drugs and leukemia.

Step -1<smiles>NNC(=O)c1cccnc1N1CCOCC1</smiles><smiles>Sc1nnc(-c2cccnc2N2CCOCC2)o1</smiles>

2-Morpholino-3-pyridinylic acid hydrazide

2-\{2-(Morpholino)-3-pyridinyl $\}-5$ mercapto-1,3,4-oxadiazole : (A)

Step -2<smiles>CC(=O)c1cc(F)c(Cl)cc1Cl</smiles>

2,4-Dichloro-5-fluoroacetophenone<smiles>[R]C=CC(=O)c1cc(F)c(Cl)cc1Cl</smiles>

Aromatic aldehyde
1-(2,4-Dichloro-5-fluoro phenyl)-3(aryl)-2-propene-1-one : (B)

Step -3

(B) +<smiles>N=C(N)N[SH](=O)([O-])[O-]</smiles>

$25 \% \mathrm{MeONa} / \mathrm{MeO}$

Reflux temp

Guanidine nitrate<smiles>[R]c1cc(-c2cc(F)c(Cl)cc2Cl)nc(N)n1</smiles>

2-Amino-4-(2,4-dichloro-5-fluoro phenyl)-6-(aryl)-pyrimidine : (C)

Step -4

(C) +<smiles>O=C(Cl)CCl</smiles>

Chloroacetyl chloride
Benzene/<smiles>CC(C)(C)[Mg][Mg]</smiles><smiles>[R]c1cc(-c2cc(F)c(Cl)cc2Cl)nc(NC(=O)CCl)n1</smiles>

N-Chloro acetyl-2-amino-4-(2,4-dichloro5-fluoro phenyl)-6-(aryl)-Pyrimidine : (D) 
Step -5

\section{Experimental}

\begin{tabular}{|c|c|c|}
\hline \multirow{11}{*}{ Where $\mathrm{R}=$} & $(\mathrm{TN}-1$ to $\mathrm{TN}-10)$ & \\
\hline & 4- $\mathrm{CH}_{3} \cdot \mathrm{C}_{6} \mathrm{H}_{4}$ & $-\mathrm{TN}-1$ \\
\hline & $4-\mathrm{N}\left(\mathrm{CH}_{3}\right)_{2} \cdot \mathrm{C}_{6} \mathrm{H}_{4}$ & - TN-2 \\
\hline & $2-\mathrm{OH} \cdot \mathrm{C}_{6} \mathrm{H}_{4}$ & $-\mathrm{TN}-3$ \\
\hline & $4-\mathrm{OH} \cdot \mathrm{C}_{6} \mathrm{H}_{4}$ & $-\mathrm{TN}-4$ \\
\hline & $4-\mathrm{Cl} \cdot \mathrm{C}_{6} \mathrm{H}_{4}$ & $-\mathrm{TN}-5$ \\
\hline & $2,4-(\mathrm{Cl})_{2} \cdot \mathrm{C}_{6} \mathrm{H}_{3}$ & - TN -6 \\
\hline & $4-\mathrm{F} \cdot \mathrm{C}_{6} \mathrm{H}_{4}$ & $-\mathrm{TN}-7$ \\
\hline & $2-\mathrm{OCH}_{3} \cdot \mathrm{C}_{6} \mathrm{H}_{4}$ & $-\mathrm{TN}-8$ \\
\hline & $4-\mathrm{OCH}_{3} \cdot \mathrm{C}_{6} \mathrm{H}_{4}$ & - TN -9 \\
\hline & $3,4,5-\left(\mathrm{OCH}_{3}\right)_{3} \cdot \mathrm{C}_{6} \mathrm{H}_{2}$ & - TN -10 \\
\hline
\end{tabular}

Step-1: Preparation of 2-\{2-(Morpholino)-3-pyridinyl $\}-5-m e r c a p t o-1,3,4-$ oxadiazole ( $A$ )

To a solution of 2-Morpholino-3-pyridinylic acid-hydrazide (0.1 mole, 22.2 g.), $\mathrm{CS}_{2}(0.1$ mole, $7.6 \mathrm{ml})$ and $20 \% \mathrm{KOH}$ solution $(10 \mathrm{ml})$ in methanol $(82 \mathrm{ml})$ was added and refluxed for eight hours. After the completion of reaction, the resultant mixture was poured in crushed ice. Product was filtered, washed with water and crystallized from ethanol to give white needles of the title compound.

Step-2: preparation of 1-(2,4-dichloro-5-fluoro phenyl)-3(aryl)-2-propene-1-one (e)

To a thoroughly stirred solution of 2,4-dichloro-5-fluoro acetophenone $(0.05$ mole, 9.7 g.) \& aromatic aldehyde (0.05 mole, 5.3 g.) in methanol $(98 \mathrm{ml}$.), was added $20 \% \mathrm{NaOH}$ solution $(10 \mathrm{ml}$.). The reaction mixture was stirred for thirty minutes at room temperature and left over night. After the completion of reaction, it was poured into ice water, acidified to neutral, filtered and crystallized from ethanol.

Step-3: preparation of 2-amino-4-(2,4-dichloro-5-fluoro phenyl)-6-(aryl)pyrimidine: $(f)$

A mixture of (E) (0.05 mole, 15.3 g.), guanidine nitrate $(0.15$ mole, 18.3 g. $)$ and sodium methoxide $(25 \%)$ in methanol (100 ml.) was refluxed for six hours. After the completion of reaction, the resultant mixture was cooled to room temperature. Separated product was filtered, washed with water, dried and crystallized from methanol. 
Step-4: preparation of n-chloro acetyl-2-amino-4-(2,4-dichloro-5-fluoro phenyl)6-(aryl)-pyrimidine ( $g$ )

In benzene $(30 \mathrm{ml})$, chloro acetyl chloride $(0.05$ mole, $5.6 \mathrm{ml})$ and 2-3 drops of TEA were added and the mixture was stirred in water bath for 10 mins. The solution of (F) (0.05 mole) in benzene $(80 \mathrm{ml})$ was added drop wise and refluxed for two hours. Then cooled the reaction mixture. The resulting white precipitates were filtered and washed with benzene, purified by recrystallization from alcohol.

Step-5: preparation of 2-[\{2-(morpholino)-3-pyridinyl-5-thio\}-2-oxoethyl oxadiazoly]]-amino-4-(2,4-dichloro-5-fluoro phenyl)-6-(aryl)-pyrimidine:

To a solution of (D) (0.005 mole) in acetone, $(\mathrm{G})(0.005$ mole) and $\mathrm{KOH}$ solution $(10 \mathrm{ml})$ in acetone $(80 \mathrm{ml})$ was added and refluxed for two hours. After the completion of reaction, the resultant mixture was poured in to crushed ice. Product was filtered, washed with water and crystallized from ethanol.

Similarly other compounds (TN-2) to (TN-10) were prepared by the above method from intermediate (D) and the corresponding N-chloro acetyl-2-amino-4-(2,4-dichloro-5-fluoro)6-(aryl)-pyrimidines $(\mathrm{G})$ and were purified by crystallization from absolute alcohol.

Table . 1 2-[\{2-(morpholino)-3-pyridinyl-5-thio \}-2-oxoethyl oxadiazolyl]-amino-4-(2,4dichloro-5-fluoro phenyl)-6-(aryl)-pyrimidines (TN-1 to TN-10)

\begin{tabular}{|c|c|c|c|c|c|c|c|c|}
\hline \multirow{2}{*}{ S.No } & \multirow{2}{*}{$\mathrm{R}$} & \multirow{2}{*}{ Mol. Formula } & \multirow{2}{*}{$\underset{\stackrel{\text { M.P. }}{{ }^{\circ} \mathrm{C}}}{ }$} & \multirow{2}{*}{$\begin{array}{l}0^{0} \\
\frac{0}{0} \\
\stackrel{0}{0}\end{array}$} & \multicolumn{4}{|c|}{ Elemental Analyses \% } \\
\hline & & & & & & $\overline{\mathrm{C}}$ & $\mathrm{H}$ & $\mathrm{N}$ \\
\hline \multirow[t]{2}{*}{ TN-1 } & \multirow[t]{2}{*}{$4-\mathrm{CH}_{3} \mathrm{C}_{6} \mathrm{H}_{4}$} & \multirow[t]{2}{*}{$\mathrm{C}_{30} \mathrm{H}_{24} \mathrm{~N}_{7} \mathrm{O}_{3} \mathrm{SFCl}_{2}$} & \multirow[t]{2}{*}{182} & \multirow[t]{2}{*}{75} & $\mathrm{R}$ & 55.22 & 3.7 & 15.07 \\
\hline & & & & & $\mathrm{F}$ & 55.18 & 3.62 & 14.99 \\
\hline \multirow[t]{2}{*}{$\mathrm{TN}-2$} & \multirow[t]{2}{*}{$4-\mathrm{N}\left(\mathrm{CH}_{3}\right)_{2} \mathrm{C}_{6} \mathrm{H}_{4}$} & \multirow[t]{2}{*}{$\mathrm{C}_{31} \mathrm{H}_{27} \mathrm{~N}_{8} \mathrm{O}_{3} \mathrm{SFCl}_{2}$} & \multirow[t]{2}{*}{162} & \multirow[t]{2}{*}{60} & $\mathrm{R}$ & 54.63 & 3.99 & 16.44 \\
\hline & & & & & $\mathrm{F}$ & 54.59 & 3.84 & 16.18 \\
\hline \multirow[t]{2}{*}{$\mathrm{TN}-3$} & \multirow[t]{2}{*}{$2-\mathrm{OH} \mathrm{C} \mathrm{C}_{6}$} & \multirow[t]{2}{*}{$\mathrm{C}_{29} \mathrm{H}_{22} \mathrm{~N}_{7} \mathrm{O}_{4} \mathrm{SFCl}_{2}$} & \multirow[t]{2}{*}{$155-157$} & \multirow[t]{2}{*}{60} & $\mathrm{R}$ & 53.22 & 3.39 & 14.98 \\
\hline & & & & & $\mathrm{F}$ & 53.17 & 3.32 & 14.93 \\
\hline \multirow[t]{2}{*}{$\mathrm{TN}-4$} & \multirow[t]{2}{*}{$4-\mathrm{OH} \mathrm{C} \mathrm{C}_{6} \mathrm{H}_{4}$} & \multirow[t]{2}{*}{$\mathrm{C}_{29} \mathrm{H}_{22} \mathrm{~N}_{7} \mathrm{O}_{4} \mathrm{SFCl}_{2}$} & \multirow[t]{2}{*}{175} & \multirow[t]{2}{*}{68} & $\mathrm{R}$ & 53.22 & 3.39 & 14.98 \\
\hline & & & & & $\mathrm{F}$ & 53.16 & 3.31 & 14.89 \\
\hline \multirow[t]{2}{*}{$\mathrm{TN}-5$} & \multirow[t]{2}{*}{ 4- $\mathrm{Cl} \mathrm{C} \mathrm{C}_{6} \mathrm{H}_{4}$} & \multirow[t]{2}{*}{$\mathrm{C}_{29} \mathrm{H}_{21} \mathrm{~N}_{7} \mathrm{O}_{3} \mathrm{SFCl}_{3}$} & \multirow[t]{2}{*}{195} & \multirow[t]{2}{*}{70} & $\mathrm{R}$ & 51.76 & 3.15 & 14.57 \\
\hline & & & & & $\mathrm{F}$ & 51.71 & 3.09 & 14.51 \\
\hline \multirow[t]{2}{*}{ TN-6 } & \multirow[t]{2}{*}{$2,4-(\mathrm{Cl})_{2} \mathrm{C}_{6} \mathrm{H}_{3}$} & \multirow[t]{2}{*}{$\mathrm{C}_{29} \mathrm{H}_{20} \mathrm{~N}_{7} \mathrm{O}_{3} \mathrm{SFCl}_{4}$} & $205-206$ & 73 & $\mathrm{R}$ & 49.26 & 2.85 & 13.86 \\
\hline & & & & & $\mathrm{F}$ & 49.20 & 2.78 & 13.81 \\
\hline $\mathrm{TN}-7$ & $4-\mathrm{F} \mathrm{C}_{6} \mathrm{H}_{4}$ & $\mathrm{C}_{29} \mathrm{H}_{21} \mathrm{~N}_{7} \mathrm{O}_{3} \mathrm{SF}_{2} \mathrm{Cl}$ & 178 & 70 & $\mathrm{R}$ & 53.09 & 3.23 & 14.94 \\
\hline & & & & & $\mathrm{F}$ & 53.02 & 3.15 & 14.86 \\
\hline TN-8 & $2-\mathrm{OCH}_{3} \mathrm{C}_{6} \mathrm{H}_{4}$ & $\mathrm{C}_{30} \mathrm{H}_{24} \mathrm{~N}_{7} \mathrm{O}_{4} \mathrm{SFCl}_{2}$ & 152 & 62 & $\mathrm{R}$ & 53.90 & 3.62 & 14.67 \\
\hline & & & & & $\mathrm{F}$ & 53.85 & 3.52 & 14.61 \\
\hline TN-9 & 4- $\mathrm{OCH}_{3} \mathrm{C}_{6} \mathrm{H}_{4}$ & $\mathrm{C}_{30} \mathrm{H}_{24} \mathrm{~N}_{7} \mathrm{O}_{4} \mathrm{SFCl}_{2}$ & 178 & 65 & $\mathrm{R}$ & 53.90 & 3.62 & 14.67 \\
\hline & & & & & $\mathrm{F}$ & 53.82 & 3.51 & 14.61 \\
\hline $\mathrm{TN}-10$ & $3,4,5-\left(\mathrm{OCH}_{3}\right)_{3} \mathrm{C}_{6} \mathrm{H}_{2}$ & $\mathrm{C}_{32} \mathrm{H}_{28} \mathrm{~N}_{7} \mathrm{O}_{6} \mathrm{SFCl}_{2}$ & 215 & 78 & $\mathrm{R}$ & 52.75 & 3.87 & 13.46 \\
\hline & & & & & $\mathrm{F}$ & 52.69 & 3.81 & 13.39 \\
\hline
\end{tabular}


IR Spectral Data<smiles>O=C(CSc1nnc(-c2cccnc2N2CCOCC2)o1)Nc1nc(-c2ccc(Cl)cc2)cc(-c2cc(F)c(Cl)cc2Cl)n1</smiles>

\begin{tabular}{|c|c|c|}
\hline \multirow{14}{*}{ IR (KBr): } & \multicolumn{2}{|r|}{ (TN-15) } \\
\hline & $1265 \mathrm{Cm}^{-1}$ & (-C-O- stretching in oxadiazole) \\
\hline & $1545 \mathrm{Cm}^{-1}$ & $(-\mathrm{C}=\mathrm{N}-$ stretching in oxadiazole $)$ \\
\hline & $1680 \mathrm{Cm}^{-1}$ & $(>\mathrm{C}=\mathrm{O}$ - stretching in amide $)$ \\
\hline & $1568 \mathrm{Cm}^{-1}$ & (-NH- deformation in amide) \\
\hline & $3248 \mathrm{Cm}^{-1}$ & (-NH stretching in amide) \\
\hline & $1245 \mathrm{Cm}^{-1}$ & (-C-O-C- stretching (asym.) in alkanyl ether) \\
\hline & $1035 \mathrm{Cm}^{-1}$ & (-C-O-C- stretching (sym.) in alkanyl ether) \\
\hline & $1472 \mathrm{Cm}^{-1}$ & (-C-H- deformation in methelene) \\
\hline & $790 \mathrm{Cm}^{-1}$ & (1,4-Disubstituted benzene) \\
\hline & $887 \mathrm{Cm}^{-1}$ & (1,2,4,5-Tetrasubstituted benzene) \\
\hline & $3168 \mathrm{Cm}^{-1}$ & (-NH- stretching in pyrimidine) \\
\hline & $2938 \mathrm{Cm}^{-1}$ & $\left(-\mathrm{S}-\mathrm{CH}_{2}-\right.$ stretching in thioether linkage) \\
\hline & $745 \mathrm{Cm}^{-1}$ & (-C-Cl- stretching in aromatic ring) \\
\hline
\end{tabular}

NMR Spectral Data<smiles></smiles>

(TN-15)

\begin{tabular}{|c|c|c|c|c|}
\hline No. & $\begin{array}{c}\text { Chemical shift } \\
\delta \mathrm{ppm}\end{array}$ & Multiplicity & $\begin{array}{l}\text { Number of } \\
\text { proton (s) }\end{array}$ & Assignment \\
\hline 1. & 3.78 & Singlet & $2 \mathrm{H}$ & $-\mathrm{CH}_{2}$ at $\underline{\mathrm{a}}$ \\
\hline 2. & 6.67 & Doublet & $1 \mathrm{H}$ & $\mathrm{Ar}-\underline{\mathrm{H}}$ at $\underline{\mathrm{b}}$ \\
\hline 3. & 6.82 & Doublet & $1 \mathrm{H}$ & $\operatorname{Ar}-\overline{\mathrm{H}}$ at $\underline{\mathrm{c}}$ \\
\hline 4. & 8.86 & Doublet & $1 \mathrm{H}$ & $\mathrm{Ar}-\overline{\mathrm{H}}$ at $\underline{\overline{\mathrm{d}}}$ \\
\hline 5. & 8.88 & Singlet & $1 \mathrm{H}$ & $-\mathrm{N} \underline{\bar{H}}$ at $\underline{\mathrm{e}}$ \\
\hline 6. & 3.87 & Triplet & $4 \mathrm{H}$ & Ar- $\overline{-H}$ at $\underline{f}$ \\
\hline 7. & 2.99 & Triplet & $4 \mathrm{H}$ & $\operatorname{Ar}-\underline{\bar{H}}$ at $\bar{g}$ \\
\hline 7. & 6.98 to 7.28 & Multiplet & $7 \mathrm{H}$ & Ar- $-\underline{H}$ at $\underline{\mathrm{h}}, \underline{\mathrm{i}}, \mathrm{j} \& \mathrm{k}$ \\
\hline
\end{tabular}




\section{Antibacterial Activity}

This part deals with the in-vitro screening of newly prepared compounds for antibacterial activity. The species S.aureus, E.coli, S.typhi and B.subtilis have been taken for the antibacterial activities. Agar-cup method was carried out for the in-vitro screening for antibacterial activity.

The results of the compounds synthesized given for antibacterial screening are mentioned in following table along with standard drugs.

Table 2 Antibacterial Activity

\begin{tabular}{|c|c|c|c|c|c|}
\hline \multirow{2}{*}{ S. No. } & \multicolumn{5}{|c|}{$\begin{array}{l}\text { (Zone of inhibition in } \mathrm{mm} \text { ) } \\
\text { at } 50 \mu \mathrm{g} / \mathrm{ml} \text { concentration }\end{array}$} \\
\hline & $\mathrm{R}$ & E.Coli & S.Aureus & S.Typhi & B.Subtilis \\
\hline TN-1 & $4-\mathrm{CH}_{3} \cdot \mathrm{C}_{6} \mathrm{H}_{4}$ & --- & --- & 09 & 10 \\
\hline $\mathrm{TN}-2$ & $4-\mathrm{N}\left(\mathrm{CH}_{3}\right)_{2} \cdot \mathrm{C}_{6} \mathrm{H}_{4}$ & 12 & 11 & 10 & 08 \\
\hline TN-3 & $2-\mathrm{OH} \cdot \mathrm{C}_{6} \mathrm{H}_{4}$ & 12 & 09 & 11 & --- \\
\hline TN-4 & $4-\mathrm{OH} \cdot \mathrm{C}_{6} \mathrm{H}_{4}$ & 10 & 09 & --- & 09 \\
\hline TN-5 & $4-\mathrm{Cl} \cdot \mathrm{C}_{6} \mathrm{H}_{4}$ & 10 & 13 & 09 & 10 \\
\hline TN-6 & $2,4-(\mathrm{Cl})_{2} \cdot \mathrm{C}_{6} \mathrm{H}_{3}$ & 12 & 13 & 12 & 09 \\
\hline TN-7 & $4-\mathrm{F} \cdot \mathrm{C}_{6} \mathrm{H}_{4}$ & 11 & 11 & --- & --- \\
\hline TN-8 & $2-\mathrm{OCH}_{3} \cdot \mathrm{C}_{6} \mathrm{H}_{4}$ & 10 & 07 & 09 & 08 \\
\hline TN-9 & $4-\mathrm{OCH}_{3} \cdot \mathrm{C}_{6} \mathrm{H}_{4}$ & 13 & 12 & 11 & 10 \\
\hline TN-10 & $3,4,5-\left(\mathrm{OCH}_{3}\right)_{3} \cdot \mathrm{C}_{6} \mathrm{H}_{2}$ & 14 & 09 & --- & --- \\
\hline Standard & Tetracycline & 15 & 19 & 24 & 21 \\
\hline Drug & Chloramphenicol & 18 & 25 & 24 & 20 \\
\hline
\end{tabular}

\section{Conclusions}

The antimicrobial screening results reveals following points. In the synthesised compounds, some compounds showed moderate to good activity against the entire microorganisms whereas some compounds were found inactive. In comparison with standard drugs compounds TN-1 \& TN-10 showed maximum zone of inhibition against E-coli., S.aureus, S.typhi and B.subtitlis. In detail the compound $\mathrm{TN}-2$ have good activity against E. coli. Compound TN-6 \& TN-10have good activity against S.Aureus while compound TN-5 \& TN-7 against S.Typhi and TN-7 against B.Subtilis have found modest activity compared to the standard drugs. The above activities are quite interesting and further study in the molecule is essential.

Thus from above discussion it may be concluded that it is worthwhile to pursue further investigation by manipulating the above novel mercapto oxadiazole derivatives. 


\section{Acknowledgement}

We acknowledge our sincere thanks to Prof. K. R. Desai, Head, Department of Chemistry, Veer Narmad South Gujarat University, Surat for valuable suggestions, inspiration and providing facilities.

\section{References}

1. Ghoneim K M and Youssef R, J Indian Chem Soc, 1986, 53, 914.

2. Kenner G W and Todd A, "Heterocyclic Compounds" Ed. R C Elderfield, Wiley, New York, 1957, 6.

3. Brown D J, "The Chemistry of Heterocyclic Compounds" Ed. A. Weissberger, Interscience, New York, 1962, 16.

4. Hunt R R, McOmie J F M and Sayer E R, J Chem Soc, 1959, 525.

5. Haley C A C and Maitland P, J Chem Soc, 1951, 3115.

6. Hale W J, J Am Chem Soc, 1914, 36,104.

7. Hunt R R, McOmie J F M and Sayer E R, J. Chem. Soc, 1959, 527.

8. Karale, B. K. and Gill, C. H. Indian J Chem, 2002, 41B, 1957.

9. Reddy V M and Sarma Rama G V S, Indian J Heterocycl Chem, 1993, 3, 111.

10. Shingare, M S, Karale B K, Gill C H, Gange K N and Bachute $\mathrm{M}$ T Indian $J$ Heterocycl Chem, 1999, 9, 153. 


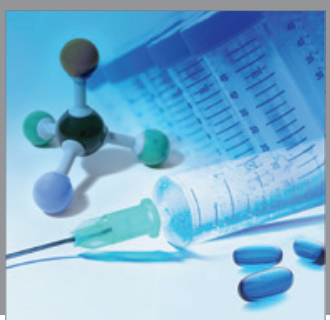

International Journal of

Medicinal Chemistry

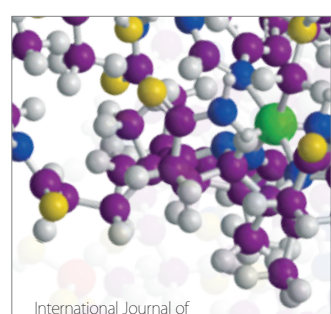

Carbohydrate Chemistry

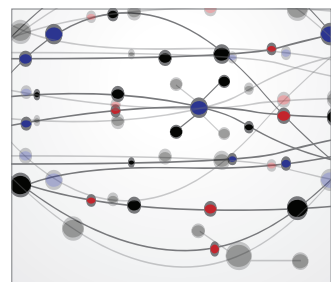

The Scientific World Journal
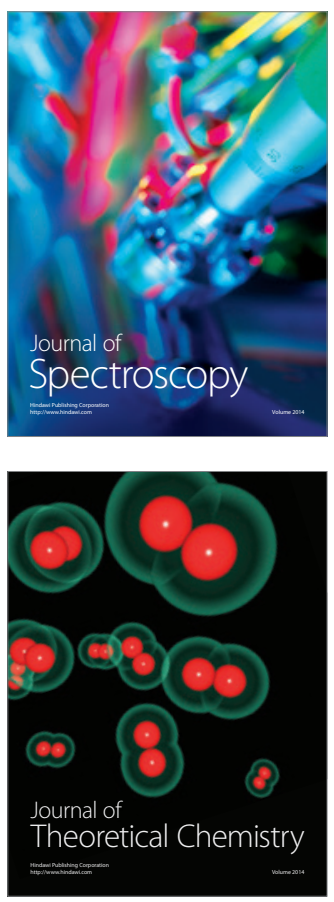
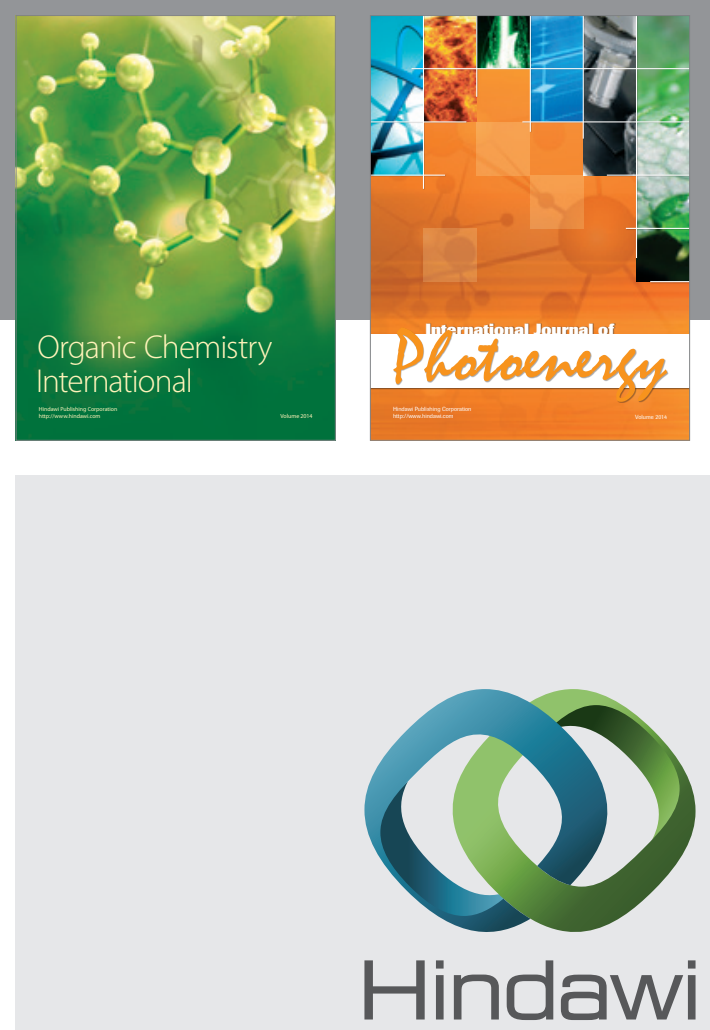

Submit your manuscripts at

http://www.hindawi.com
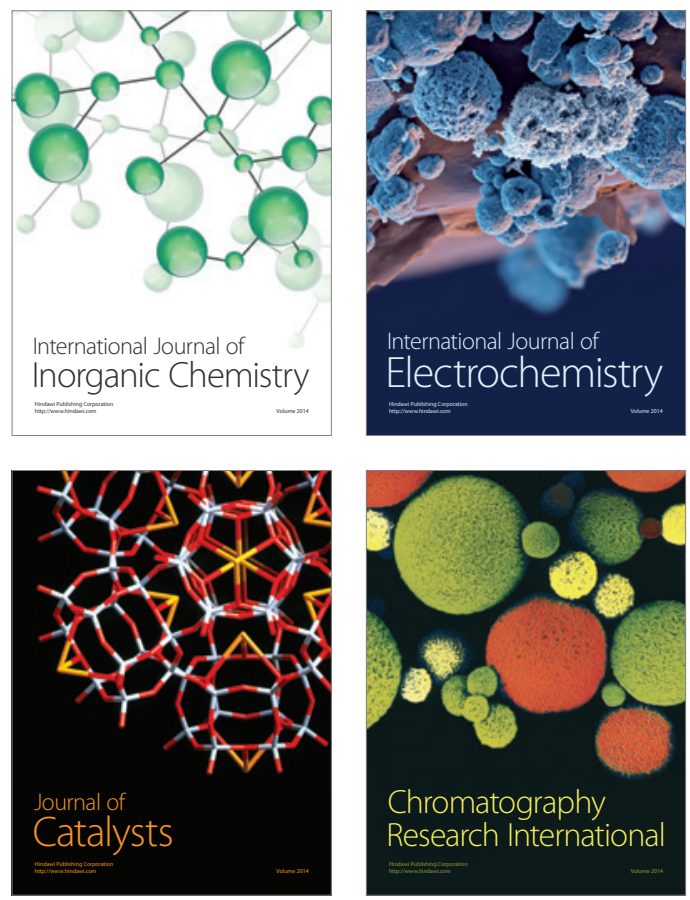
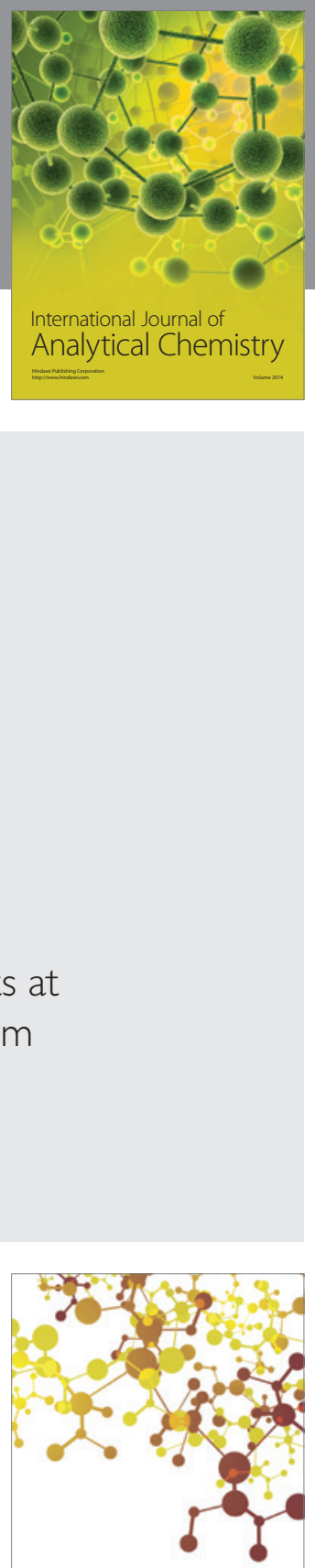

Journal of

Applied Chemistry
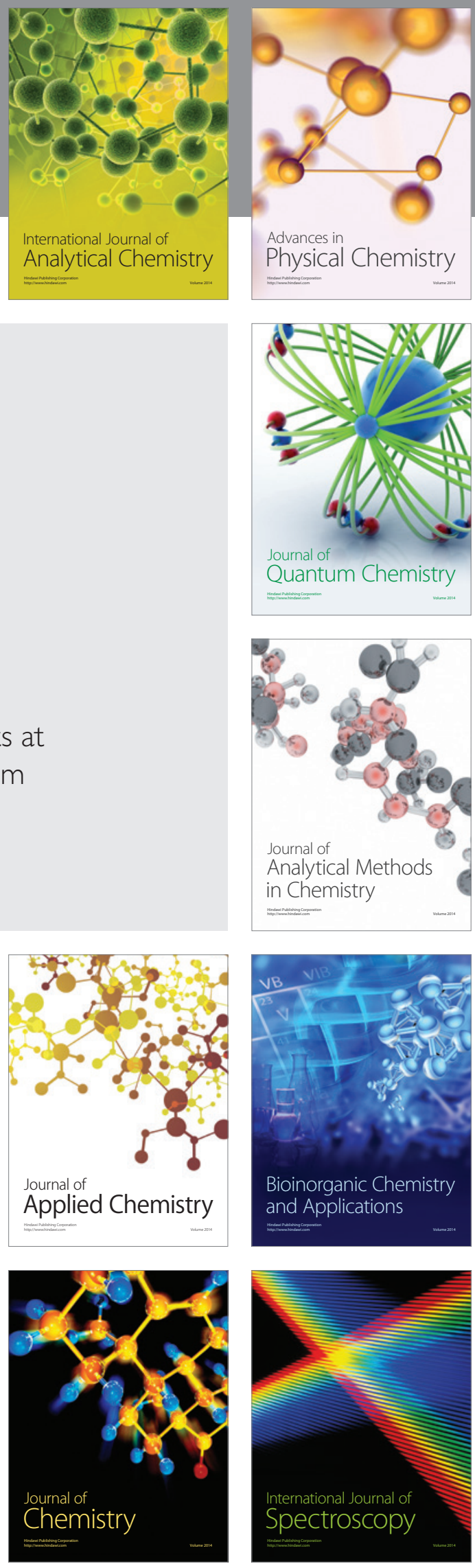